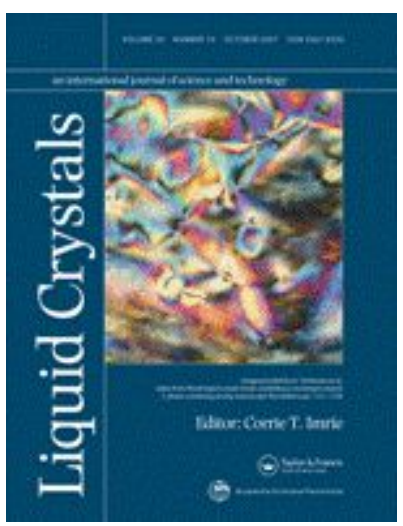

\title{
NMR of quadrupole noble gases in liquid crystals
}

\begin{tabular}{|r|l|}
\hline Journal: & Liquid Crystals \\
\hline Manuscript ID & TLCT-2019-0094.R2 \\
\hline Manuscript Type: & Special Issue Article \\
\hline Date Submitted by the \\
Author: & n/a \\
\hline Keywords: & $\begin{array}{l}\text { Quadrupolar noble gases, Electric field gradient, Second order } \\
\text { coupling } \\
\text { counte shift, Biaxiality, Orientational order, Tilt angle, Hexadecapole }\end{array}$ \\
\hline &
\end{tabular}

\section{SCHOLARONE Manuscripts}




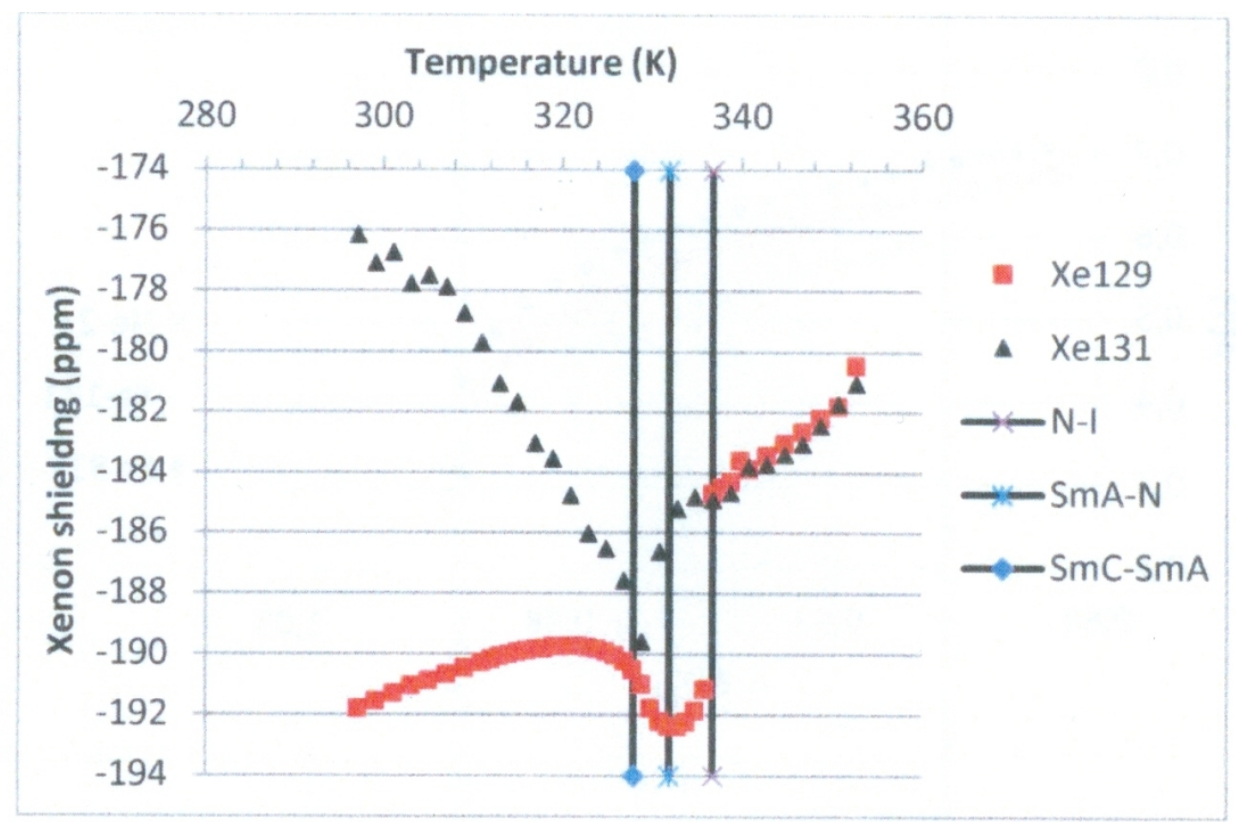

$115 \times 74 \mathrm{~mm}(300 \times 300$ DPI $)$ 


\title{
NMR of quadrupole noble gases in liquid crystals
}

\author{
Jukka Jokisaari* \\ NMR Research Unit, University of Oulu, P.O.Box 3000, 90014 University of Oulu, Finland
}

\begin{abstract}
NMR spectroscopy of quadrupolar noble gases, ${ }^{21} \mathrm{Ne}(\operatorname{spin} 3 / 2),{ }^{83} \mathrm{Kr}(9 / 2),{ }^{131} \mathrm{Xe}(3 / 2)$, dissolved in thermotropic liquid crystals enables the derivation of versatile information about the physical properties of the materials. The spectra display fine structures, triplets and nonets, which reveal quadrupole couplings. These in turn can be used in the determination of electric field gradients (EFG), orientational order parameters, and tilt angles. Comparison of ${ }^{129} \mathrm{Xe}$ and ${ }^{131} \mathrm{Xe}$ chemical shifts reveals second order quadrupole shifts (SOQS) in circumstances where the ratio of quadrupole coupling and magnetic flux density is suitable. The ${ }^{131} \mathrm{Xe}$ SOQS is shown to distinguish between uniaxial and biaxial nematic phases. Both ${ }^{83} \mathrm{Kr}$ and ${ }^{131} \mathrm{Xe}$ NMR spectra may display asymmetry around the central transition because of the SOQS, which can be used to identify biaxiality in nematic phases. As a curiosity, the effect of possible hexadecapole coupling on the ${ }^{83} \mathrm{Kr}$ NMR transitions of krypton in liquid crystals is discussed.
\end{abstract}

\section{Keywords}

Quadrupolar noble gases, electric field gradient, orientational order, second order quadrupole shift, biaxiality, tilt angle, hexadecapole coupling

* Email: Jukka.Jokisaari@oulu.fi

ORCID 0000-0003-2190-1775 


\section{Introduction}

NMR active noble gases, ${ }^{21} \mathrm{Ne}(\operatorname{spin} 3 / 2),{ }^{83} \mathrm{Kr}(9 / 2),{ }^{129} \mathrm{Xe}(1 / 2)$ and ${ }^{131} \mathrm{Xe}(3 / 2)$, are practical probes in studies of various materials. Their NMR chemical shifts and quadrupolar interactions stem exclusively from environmental effects. Table 1 lists their properties from the NMR point to view. Apart from the quadrupolar noble gases, ${ }^{129} \mathrm{Xe}$ NMR $\left({ }^{129} \mathrm{Xe}\right.$ spin $\left.=1 / 2\right)$ has appeared a means to derive versatile information about liquid crystals [1-3]. It is a practical nucleus for many reasons: its spin is $1 / 2$, its receptivity is over 30 -fold compared to that of ${ }^{13} \mathrm{C}$, and its electron cloud is easily polarized by environmental contributions. Interactions in liquid-crystalline solutions deform the electron cloud of xenon resulting in anisotropic shielding tensor [4,5]. On the other hand, this deformation causes an electric field gradient (EFG) which together with EFG created by quadrupole moments of liquid crystals molecules leads to a triplet structure in ${ }^{131} \mathrm{Xe}$ and ${ }^{21} \mathrm{Ne}$ NMR spectra and a nonet structure in ${ }^{83} \mathrm{Kr}$ NMR spectra. The ${ }^{131} \mathrm{Xe}$ chemical shift behaves in the same way as that of ${ }^{129} \mathrm{Xe}$ in isotropic and uniaxial liquid crystal phases but differs in tilted and biaxial phases.

\section{Table 1 here}

\section{Experimental}

The liquid crystals mentioned in the text are commercial products: ZLI 1167 from Merck and FELIX-R\&D from Hoechst. The composition of the latter has not been revealed by the manufacturer and therefore it was done in Ref. [9] applying multinuclear NMR spectroscopy and sophisticated computer analysis. As Table 1 indicates, the natural abundance and receptivity of ${ }^{21} \mathrm{Ne}$ are very low. Therefore, ${ }^{21} \mathrm{Ne}$ enriched (degree of enrichment 95 at. \%, from Isotec Inc. USA) gas was used. Also, the ${ }^{83} \mathrm{Kr}$ experiments were performed from ${ }^{83} \mathrm{Kr}$ enriched gas $(74.8$ at. \%, from Isotec Inc. USA). In order to reach a reasonable signa-to-noise ratio in the spectra accumulation time of up to 3 hours for ${ }^{21} \mathrm{Ne}$ and up to ca. 8 hours for ${ }^{83} \mathrm{Kr}$ per temperature was needed. The reason for the long time in the latter case is not only the low receptivity but also the distribution of 
intensity among 9 resonance lines. The experiment time varied from 1 hour to 4 hours per temperature when using natural ${ }^{131}$ Xe gas. Experiments were carried out on Bruker DSX300WB, Bruker DPX400, Bruker DRX400 and Bruker Avance III 600 spectrometers. The studied samples were made generally into $10-\mathrm{mm}$ heavy-wall NMR tubes. However, the ${ }^{131} \mathrm{Xe}$ experiments on the $600 \mathrm{MHz}$ instrument were done from a 5-mm heavy-wall (wall thickness $1 \mathrm{~mm}$ ) tube

\section{3, Results and discussion}

\subsection{Determination of the orientational order parameter and electric field gradient}

Measurement of the quadrupole coupling (QC) of a quadrupolar noble gas allows for the determination of the temperature dependence of orientational order parameters as well as the total electric field gradient (EFG) at the nuclear site. Here we consider exclusively the conventional second rank order parameter $S(T)$ in a nematic phase, although the order parameters of smectic phases can be obtained as well [10]. The QC, $\left|\Delta_{\mathfrak{v} \mid}\right|$, experienced by a quadrupolar noble gas nucleus can be presented in the form [9]

$$
\left|\Delta_{V}\right|=|\chi|\left|i P_{2}(\cos \theta)\right|=\left|\frac{e Q_{i}\left(F_{|l|}^{\mathrm{tot}}\right\rangle}{h}\left(1-\gamma_{\infty i}\right) P_{2}(\cos \theta)\right|=\left|\frac{e Q_{i}}{h} P_{2}(\cos \theta)\left(1-\gamma_{\infty i}\right)\left(A_{i}+B_{i \frac{T}{T_{N I}}}\right) S(T)\right|
$$

where $\chi \| \boldsymbol{i}$ is the quadrupole coupling tensor element in the direction of the liquid crystal director, $e Q_{i}$ is the electric nuclear quadrupole moment, $\left\langle\boldsymbol{F}_{\| \boldsymbol{i}}^{\text {tot }}\right\rangle$ is the average total EFG in the direction of the liquid crystal director, $\boldsymbol{\gamma}_{\infty \boldsymbol{i}}$ is the Sternheimer antishielding factor [7] and $\theta$ is the angle between the external magnetic field and the liquid crystal director. In the following, we treat, as an example, neon-21, krypton-83 and xenon-131 in thermotropic liquid crystal Merck ZLI 1167 [11]. This liquid crystal possesses negative diamagnetic anisotropy, and consequently its director orients perpendicularly to the external magnetic field. The total EFG at the nuclear site constitutes two 
contributions; one from the LC molecules and the other one from the deformation of the electron cloud of the noble gas atom [10]. $A_{i}$ and $B_{i}$ refer to these contributions [9]. The orientational order parameter is modelled by the Haller function [12]

$S(T)=\left(1-y \frac{T}{T_{N I}}\right)^{z}$

Figure 1 shows the magnitude (spectra do not reveal the sign) of experimental ${ }^{21} \mathrm{Ne}$ quadrupole coupling together with the calculated curve obtained from least-squares fit of function (1) to the experimental points. The adjustable parameters are $A, B$ and $z$ while $y$ is fixed to the value 0.998 [13]. The corresponding results obtained for krypton-83 are displayed in Fig. 2 and for xenon-131 in Fig. 3. Table 2 lists the values of the variables. The appearance of a maximum in each curve proves that the $A$ and $B$ factors are of opposite sign. One should note that the amount of dissolved gas affects to some extent the phase transition temperatures.

\section{Figure 1 here}

Figure 2 here

Figure 3 here

Table 2 here

The orientational order parameters, $S(T)$, derived from the least-squares fits are illustrated in Fig. 4.

\section{Figure 4 here}

\subsection{Second order quadrupole shift, phase biaxiality and tilt angle}

Figure 5 displays the comparison of the ${ }^{129} \mathrm{Xe}$ and ${ }^{131} \mathrm{Xe}$ shielding as a function of temperature in the ferroelectric liquid crystal FELIX-R\&D [14], which possesses, among the isotropic phase, three 
liquid-crystalline phases: nematic $\mathrm{N}^{*}$, smectic $A$ and smectic $\mathrm{C}^{*}$. (In strong magnetic fields, the helical structure unwinds.) The electronic structure of the two xenon isotopes is the same, and consequently their shielding should follow the same temperature dependence. Figure 5 shows that, unexpectedly, this is not the case in the liquid-crystalline phases of FELIX-R\&D. The ${ }^{131} \mathrm{Xe}$ NMR spectrum in liquid-crystalline solutions is a triplet (contrary to a singlet in the isotropic phase) because of the quadrupole interaction. Consequently, the different behaviour obviously stems from the (strong) ${ }^{131} \mathrm{Xe}$ quadrupole coupling.

\section{Figure 5 here}

Let us consider first the SmC phase of FELIX-R\&D, which is a tilted phase. The quadrupole Hamiltonian for a cylindrically symmetric quadrupole coupling tensor, and thus for a quadrupolar nucleus in a uniaxial liquid crystal, is (in frequency units) [16]

$\hat{H}_{Q}=\frac{e Q\left\langle V_{\|}\right\rangle}{4 I(2 I-1) h}$

$\left\{\frac{1}{2}\left(3 \cos ^{2} \theta-1\right)\left[3 \hat{I}_{z}^{2}-I(I+1)\right]+\frac{3}{2} \sin \theta \cos \theta\left[\hat{I}_{z}\left(\hat{I}_{+}+\hat{I}_{-}\right)+\left(\hat{I}_{+}+\hat{I}_{-}\right) \hat{I}_{z}\right]+\quad \frac{3}{2} \sin ^{2} \theta\left(\hat{I}_{+}^{2}+\hat{I}_{-}^{2}\right)\right\}$

where $\left\langle V_{\|}\right\rangle=e q$ is the principal component of the EFG tensor in the direction of the liquid crystal director, $e Q$ is the electric quadrupole moment of the nucleus $\left(Q=-114.6(1.1) \times 10^{-31} \mathrm{~m}^{2}\right.$ for xenon131 Ref. [6], and $e$ is the positive elementary charge) and $\theta$ is the angle between the director and the external magnetic field $\mathbf{B}_{\mathrm{o}}$, which is taken to be along the $\mathrm{z}$ axis of the laboratory frame. The spin operators have their usual meaning, i.e. $\hat{I}_{z}$ is the $\mathrm{z}$ component of the nuclear spin angular momentum operator and $\hat{I_{+}}=\frac{1}{2}\left(\hat{I_{x}}+i \hat{I_{y}}\right)$ and $\hat{I_{-}}=\frac{1}{2}\left(\hat{I_{x}}-i \hat{I_{y}}\right)$ are the raising and lowering operator, respectively. Considering a spin-3/2 nucleus, such as xenon-131, and using the operator (2) as a perturbation to the Zeeman Hamiltonian 
$\hat{H}_{Z}=-v_{L} \hat{I}_{Z}$

where $v_{L}=\left(\frac{\gamma B_{o}}{2 \pi}\right)(1-\sigma)=v_{o}(1-\sigma)$ and $\gamma$ is the gyromagnetic ratio, the transition frequencies up to second order are derived and are listed in Table 3.

\section{Table 3 here}

Table 3 shows that the second order correction to the resonance frequency of the central peak of the ${ }^{131}$ Xe triplet is (in $\left.\mathrm{Hz}\right)$

$$
\text { vSOQS }=-\frac{3 \chi^{2}}{64 u}\left(1-\cos ^{2} \theta\right)\left(9 \cos ^{2} \theta-1\right) .
$$

The angle dependent part is positive when $\theta<$ ca. $70.4^{\circ}$ and smaller than 2 . Consequently, we can make a rough estimate that $v_{S O Q S}$ becomes observable when $\frac{3 \chi^{2}}{64 n} \geq \frac{n / 2}{\sqrt{S / N}}$, where $v_{1 / 2}$ is the full width at half height $(\mathrm{FWHH})$ and $S / N$ the signal-to-noise ratio of the ${ }^{131} \mathrm{Xe}$ central transition. It is estimated that $\chi$ should be bigger than about $100 \mathrm{kHz}$ at $\mathrm{B}_{\mathrm{o}}=7.05 \mathrm{~T}$.

Because of SOQS the increase of the ${ }^{131}$ Xe shielding is (in ppm)

$$
\sigma_{S O Q S}=\frac{3}{64}\left(\frac{\chi}{n}\right)^{2}\left(1-\cos ^{2} \theta\right)\left(9 \cos ^{2} \theta-1\right)
$$

The experimental ${ }^{131} \mathrm{Xe}$ second order shifts in the smectic C phase of FELIX-R\&D are displayed in Figure 6. As can be seen, the shift increases from 0 to $16 \mathrm{ppm}$ when moving from $c a .330 \mathrm{~K}$ to $c a$. $297 \mathrm{~K}$. 


\section{Figure 6 here}

The distance of the satellite transitions, $\Delta$, in the ${ }^{131}$ Xe NMR spectrum is (see Fig. 7)

$$
\Delta=\left|\chi P_{2}(\cos \theta)\right|=\left|\frac{\chi}{2}\left(3 \cos ^{2} \theta-1\right)\right|
$$

and thus

$$
\chi=\left|\frac{2 \Delta}{3 \cos ^{2} \theta-1}\right| .
$$

The second order correction to the shielding becomes exclusively the function of the tilt angle $\theta$ and experimentally obtainable $\Delta$, as shown in Eqn. (8).

\section{Figure 7 here}

OSOQS $=\frac{3}{16}\left(\frac{\Delta}{v_{L}}\right)^{2} \frac{-9 \cos ^{4} \theta+10 \cos ^{2} \theta-1}{9 \cos ^{4} \theta-6 \cos ^{2} \theta+1}, \cos \theta \neq \pm \frac{1}{\sqrt{3}}$.

Application of Eqn. (8) to the case shown in Figs. 5 and 6 leads to the temperature dependent tilt angle in fair agreement with the values determined by applying electric field and optical detection, as displayed in Fig. 8.

\section{Figure 8 here}

From Table 3, one can conclude that the ${ }^{131}$ Xe triplet is asymmetric in the second order. In other words, the satellite transitions are at different distances from the central transition.

\section{3. ${ }^{131} \mathrm{Xe}$ SOQS and biaxial nematic phase}


Figure 5 indicates that ${ }^{129} \mathrm{Xe}$ and ${ }^{131} \mathrm{Xe}$ shieldings do not differ only in the smectic $\mathrm{C}$ phase but also in the nematic phase. In the nematic phase, the director is oriented along the external magnetic field, and thus $\theta=0$. This means that the theory described above cannot be applied to the nematic phase because osoQs would be zero, as can be concluded from Eqn. (4) and experiments performed in a uniaxial liquid crystal [15]. In a biaxial nematic phase, the quadrupole Hamiltonian operator is (in frequency units) [16]

$\hat{H}_{Q}=\frac{e^{2} q Q}{4 I(2 I-1) h}\left[3 \hat{I}_{z}^{2}-I(I+1)+\frac{1}{2} \eta\left(\hat{I}_{+}^{2}+\hat{I}_{-}^{2}\right)\right]$

where $\eta=\left(\chi_{\mathrm{xx}}-\chi_{\mathrm{yy}}\right) / \chi_{\mathrm{zz}}$ is the asymmetry parameter of the quadrupole coupling tensor and $e^{2} q Q / h=$ $\chi_{z z}$ is the component of the quadrupole coupling tensor in the direction of the LC director (which in the present case is along the external magnetic field). For a spin-3/2 system, such as ${ }^{131} \mathrm{Xe}$, Eqn. (9) becomes

$\hat{H}_{Q}=\frac{\chi_{z z}}{12}\left[3 \hat{I}_{z}^{2}-\frac{15}{4}+\frac{1}{2} \eta\left(\hat{I}_{+}^{2}+\hat{I}_{-}^{2}\right)\right]$

Including the Zeeman interaction and applying the second order perturbation theory as above the transition frequencies in the ${ }^{131} \mathrm{Xe}$ NMR spectrum are obtained. They are collected in Table 4.

\section{Table 4 here}

The central transition shifts in the second order by

$$
v_{S O Q S}=\frac{1\left(\eta \chi_{z z}\right)^{2}}{48 v}
$$


compared to that in the first order. The shift $v_{S O Q S}$ is always positive and differs from zero only if the ${ }^{131} \mathrm{Xe}$ quadrupole coupling tensor is asymmetric, i.e. $\eta \neq 0$. It is well known that the electron cloud of xenon-129 deforms in a uniaxial nematic phase so that its shielding tensor becomes anisotropic, $\Delta \sigma=\sigma_{\|}-\sigma_{\perp} \neq 0$, where subscripts $\|$ and $\perp$ refer to the tensor elements in the direction of the director and perpendicularly to it [4,5]. A plausible conclusion is that the quadrupole coupling tensor becomes asymmetric in a biaxial nematic phase because the EFG tensor is no more axially symmetric. As was noted above, part of the EFG arises from the deformation of the electron cloud of an atom.

The ${ }^{131} \mathrm{Xe}$ triplet becomes asymmetric in the second order, as in the smectic $\mathrm{C}$ phase discussed above, and the second order quadrupole shift is always toward increasing frequency. In the shielding scale, the ${ }^{131} \mathrm{Xe}$ SOQS becomes

$\underset{\operatorname{biax}}{\operatorname{OSOQS}}=-\frac{1}{48}\left(\frac{\chi x x-\chi y y}{\nu}\right)^{2}$

In the nematic phase of FELIX-R\&D $\stackrel{\text { biax }}{\text { bia }}$ equals $-7.062 \mathrm{ppm}$ at $334.9 \mathrm{~K}$ and $-7.192 \mathrm{ppm}$ at 332.9 K. These second order shifts result in the $\chi x x-\chi y y$ values of 455.5 and $459.7 \mathrm{kHz}$, respectively. At $334 \mathrm{~K}, \chi_{z z}=511 \mathrm{kHz}[18]$, and consequently $\eta \approx 0.89$. Thus the ${ }^{131} \mathrm{Xe}$ quadrupole coupling tensor is remarkably asymmetric in the nematic phase of FELIX-R\&D, reflecting significant biaxiality of the phase. The quadrupole coupling tensor is traceless and thus it is possible, with the knowledge above, to determine all three diagonal elements of the tensor. They are the following: $\chi_{z z}= \pm 511$ $\mathrm{kHz}, \chi_{y y}=\mp 484.5 \mathrm{kHz}$ and $\chi_{x x}=\mp 26.5 \mathrm{kHz}$. Experimental spectrum does not reveal the sign of the quadrupole coupling and therefore \pm and $\mp$ signs.

According to Eqns. (8) and (12), the ${ }^{131} \mathrm{Xe}$ SOQS is inversely proportional to the square of magnetic flux density $\left(\boldsymbol{\nu}_{\mathrm{L}} \sim B_{\mathrm{o}}\right)$. This is illustrated in Fig. 9. 


\section{Figure 9 here}

Figure 10 shows the ${ }^{131} \mathrm{Xe}$ SOQS as a function of the square of magnetic flux density. Linear leastsquares fit allows for the determination of $\chi x x-\chi y y$ which appears to be $458.6 \mathrm{kHz}$ at $332.9 \mathrm{~K}$ in perfect agreement with the value reported above.

\section{Figure 10 here}

Asymmetry of the ${ }^{131} \mathrm{Xe}$ quadrupole coupling tensor is obtained also by taking the difference of the distances of the satellites from the central peak. Using the frequencies in Table 3 the distances differ by

$$
D=\frac{1(\chi x x-\chi y y)^{2}}{24 \quad v_{L}}
$$

To estimate whether $D$ is measurable, let $\chi^{x x}-\chi y y$ equal $458 \mathrm{kHz}$ (see above) and $v_{L} 24.742 \mathrm{MHz}$. With these values $D$ is ca. $350 \mathrm{~Hz}$, most likely observable although the resonance lines are relatively broad.

Difference in the ${ }^{129} \mathrm{Xe}$ and ${ }^{131} \mathrm{Xe}$ shieldings is none-zero also in the smectic A phase (see Figs. 5 and 9), in which the director is along the external magnetic field as in the nematic phase. This means that the same theory as applied to the nematic phase is applicable here, too. For example, at $331 \mathrm{~K}$ the difference, in other words the SOQS, is $-5.6 \mathrm{ppm}$. This leads to the anisotropy of the ${ }^{131} \mathrm{Xe}$ quadrupole coupling tensor $\chi x x-\chi y y$ of $405 \mathrm{kHz}$. The tensor component $\chi_{z z}$ is ca. $450 \mathrm{kHz}[9]$ at the temperature in question and thus the asymmetry parameter $\eta=0.9$, very similar with the value derived in the nematic phase.

\section{4. ${ }^{83} \mathrm{Kr} S O Q S$}


The nuclear spin of krypton-83 is $9 / 2$ which means that the ${ }^{83} \mathrm{Kr}$ NMR spectrum consists of 9 peaks in a liquid-crystalline solution as shown in Fig. 11. Distance between two consecutive peaks is $\chi / 24$ ( $\chi$ is the quadrupole coupling constant) when neglecting the second order effects and possible hexadecapole coupling, which will be discussed below. (One should notice that in the case of nuclei with spin $3 / 2$, the respective distance is $\chi / 2$.) Application of the same procedure as above leads to the ${ }^{83} \mathrm{Kr}$ SOQS of the central transition $(-1 / 2 \ldots+1 / 2)$

$$
\underset{\operatorname{biax}}{\operatorname{OSOQS}}=-\frac{1}{864}\left(\frac{\chi x x-\chi y y}{u}\right)^{2}
$$

where $\chi x x-\chi y y$ is the asymmetry of the ${ }^{83} \mathrm{Kr}$ quadrupole coupling tensor and $v L(11.548 \mathrm{MHz}$ at $7.05 \mathrm{~T}$ ) is the ${ }^{83} \mathrm{Kr}$ Larmor frequency. In order to see how this compares with the corresponding ${ }^{131} \mathrm{Xe}$ shift, knowledge of the ${ }^{83} \mathrm{Kr}$ quadrupole coupling tensor asymmetry should be available. Unfortunately, this is not the case. Second order shifts of the other transitions are discussed below and shown in Tables 5 and 6.

\section{Figure 11 here}

\section{5. ${ }^{83} \mathrm{Kr}$ hexadecapole coupling}

A nucleus with spin $\geq 2$ possesses apart from the electric quadrupole moment also an electric hexadecapole moment. Consequently, krypton-83 with spin $9 / 2$ belongs to this class $[19,20]$. Attempts to reveal the hexadecapole coupling by NMR are practically in all cases concentrated on solid samples. In these cases, however, the strong quadrupole coupling and wide resonance lines 
may mask the weak hexadecapole coupling. Therefore, endeavors focus to get rid of the quadrupole coupling using crystals with favorable symmetry and orientation with respect to the magnetic field, and application of multi-pulse NMR methods for line narrowing [21]. NMR experiments carried out in liquid-crystalline solutions may not suffer about such problems [22]. This is because the hexadecapole interaction simply causes an additional effect on the distances between consecutive resonance peaks in a quadrupole coupled spectrum. This is discussed below.

The Hamilton operator of the hexadecapole interaction for ${ }^{83} \mathrm{Kr}$ in a liquid-crystalline solution, in which the director is along the external magnetic field, is (in frequency units) [21]

$\hat{H}=\frac{\chi^{H}}{290304}\left(35 \hat{I}_{z}^{4}-\frac{1435}{2} \hat{I}_{z}^{2}+\frac{27027}{16}\right)$,

where $\chi H=\frac{e^{2} H V_{z z z z}}{h}$ is the hexadecapole coupling constant with $e$ positive elementary charge, $H$ hexadecapole moment, and $V_{z z z z}$ gradient of the electric field gradient. The energies corresponding to the three interactions, Zeeman, quadrupole and hexadecapole, together with the second order corrections are given in Table 5. Transition frequencies in turn are in Table 6.

\section{Table 5 here}

Table 6 here

We can now conclude from Table 6 that

(a) if the spectral fine structure arises exclusively from the first order quadrupole coupling, the frequency separation of each consecutive peak is $6 \mathrm{~A}=\chi / 24$,

(b) if the hexadecapole coupling is present, and second order corrections are neglected, the separation increases or decreases, depending on the sign of the hexadecapole coupling, toward the edges of the spectrum. This is shown in Table 7, 
(c) if the quadruple coupling is large and hexadecapole coupling is neglected, the spectrum displays asymmetry around the central transition because of the SOQS. This is shown in Table 7, too.

\section{Table 7 here}

From Table 7, we may conclude that the distances of the transitions 4 and 6 from the central transition 5 differ by $4 \mathrm{C}=4 \times 12\left[\left(\chi_{\mathrm{xx}}-\chi_{\mathrm{yy}}\right) / 144\right]^{2} / \boldsymbol{v}_{\mathrm{L}}=\left(\chi_{x x}-\chi_{y y}\right)^{2} / 432 v_{L}$. Therefore, similarly to the ${ }^{131} \mathrm{Xe}$ case, the ${ }^{83} \mathrm{Kr}$ NMR spectrum reveals the asymmetry of the respective quadrupole coupling tensor, and evidently the biaxiality of the environment.

The spectrum shown in Fig, 11 was taken in a uniaxial liquid crystal. This means that the second order corrections vanish. Thus it is principle possible to determine the hexadecapole coupling. Unfortunately, the uncertainty in transition frequencies did not allow it.

\section{Conclusions}

NMR spectroscopy of quadrupolar noble gases dissolved in thermotropic liquid crystals is a valuable means to obtain information on phase transitions, orientational order parameters, electric field gradients, tilt angles and biaxiality. Particularly, the determination of biaxiality is straight forward because the experiments can be performed from static samples. A successful experiment requires, however, large enough quadrupole coupling and/or low enough magnetic flux density. A disadvantage in the ${ }^{83} \mathrm{Kr}$ and ${ }^{131} \mathrm{Xe}$ NMR experiments may be the long experiment time, as usually sufficiently low magnetic flux density is required for the observation of the asymmetry of the spectra. On the other hand, parallel use of ${ }^{129} \mathrm{Xe}$ and ${ }^{131} \mathrm{Xe}$, when identifying biaxial nematic phases, does not suffer from such a problem because for ${ }^{131} \mathrm{Xe}$ only the central resonance peak must be detected. 


\section{Acknowledgments}

The author thanks his colleagues in the former NMR research group, now NMR Research Unit in the University of Oulu for long-lasted co-operation. Dr. L.P. Ingman is tanked for the ${ }^{21} \mathrm{Ne},{ }^{83} \mathrm{Kr}$ and ${ }^{131} \mathrm{Xe}$ NMR experiments in ZLI 1167 and Dr. A.M. Kantola for the ${ }^{83} \mathrm{Kr}$ NMR spectrum displayed in Fig.11.

\section{References}

[1] Jokisaari J, NMR of noble gases dissolved in liquid crystals. In: Burnell EE, de Lange CA, editors. NMR of Ordered Liquids, Dordrecht, Kluwer Academic Publishers, 2003. p. 109-135.

[2] Jokisaari J, Noble gas probes in NMR studies of liquid crystals. In: Dong RY, editor. Nuclear Magnetic Resonance Spectroscopy of Liquid Crystals, Singapore, World Scientific, 2010. p. 59116.

[3] Jokisaari J, Luckhurst GR, Timimi B, et al. Twist-bend nematic phase of the liquid crystal dimer CB7CB: orientational order and conical angle determined by ${ }^{129} \mathrm{Xe}$ and ${ }^{2} \mathrm{H} \mathrm{NMR}$ spectroscopy, Liq. Cryst. 2015;42:708-721.

[4] Jokisaari J, Diehl P, NMR study of the shielding of the ${ }^{129} \mathrm{Xe}$ isotope of xenon gas dissolved in thermotropic liquid crystals, Liq. Cryst. 1990;7:739-748.

[5] Münster O, Jokisaari J, Diehl P, NMR determination of the ${ }^{129}$ Xe shielding anisotropy for xenon gas dissolved in liquid crystals, Mol. Cryst. Liq. Cryst. 1992; 206:179-186.

[6] Pyykkö P, Year-2017 nuclear quadrupole moments, Molec. Phys. 2018;116;1328-1338.

[7] Sternheimer RM, Effect of atomic core on nuclear quadrupole coupling, Phys. Rev.1954; 95:736-750.

[8] Kolb D, Johnson WR, Shorer P, Electric and magnetic susceptibilities and shielding factors for closed-shell atoms and ions of high nuclear charge, Phys. Rev. A 1982;26:19-31.

[9] Ahola SK, Ingman P, Laatikainen R, et al. ${ }^{21} \mathrm{Ne}$ and ${ }^{131} \mathrm{Xe}$ NMR study of electric field gradients and multinuclear NMR study of the composition of a ferroelectric liquid crystal, J. Chem. Phys. 2018;149:234901.

[10] Lounila J, Münster O, Jokisaari J, et al. Temperature-dependence of nuclear shielding and 
quadrupole coupling of noble gases in liquid crystals, J. Chem. Phys. 1992; 97:8977-8985.

[11] ZLI 1167 consists of 36\% 4-n-propyl-trans, trans-bicyclohexyl-4'-carbonitrile, 34\% 4-npentyl-trans,trans-bicyclohexyl-4'-carbonitrile, and 30\% 4-n-heptyl-trans, trans-bicyclohexyl4'-carbonitrile).

[12] Haller I, Thermodynamic and static properties of liquid crystals, Progr. Solid State Chem. 1975;10:103-118.

[13] Buka A, de Jeu WH, Diamagnetism and orientational order of nematic liquid crystals, J. Phys. 1982;43:361-367.

[14] FELIX-R\&D is a mixture of three phenyl pyrimidines. For more details, see Ref. [9].

[15] Jokisaari JP, Kantola AM, Lounila JA, et al. Detection of phase biaxiality in liquid crystals by use of the quadrupole shift in ${ }^{131}$ Xe NMR spectra, Phys. Rev. Lett. 2011;106:017801.

[16] Abragam A, The Principles of Nuclear Magnetism, Oxford University, Oxford, 1961.

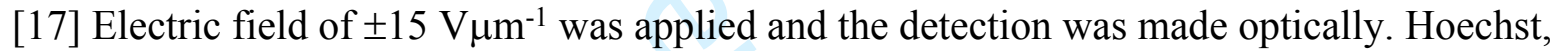
Ferroelectric liquid crystal mixtures. Data sheet. June 1995.

[18] Jokisaari J, Zhu J, Xenon NMR of phase biaxiality in liquid crystals, Magn. Reson. Chem. 2014;52:556-559.

[19] Mahler RJ, James LW, Nuclear electric hexadecapole-phonon interaction, Proc. XIV Colloque Ampere, Sept. 6-11, 1966, Ljubljana.

[20] Mahler RJ, Nuclear hexadecapole interactions, Phys. Rev.1966;152:325-330.

[21] Doering EB, Waugh JS, Search for hexadecapole interaction in $\mathrm{KTaO}_{3}$ by ${ }^{181} \mathrm{Ta}-\mathrm{NMR}$, J. Chem. Phys. 1986;85:1753-1756.

[22] Tracey AS, Radley K, Nuclear electric hexadecapole coupling in the caesium cation. Liq. Cryst. 1989;6:319-323. 
Magnitude of the $21 \mathrm{Ne}$ quadrupole coupling as a function of temperature in ZLI 1167 liquid crystal. This particular liquid crystal possesses smectic $A$ phase at the lower temperatures. Therefore, a few points near the phase transition were omitted from the least-squares fit.

$120 \times 61 \mathrm{~mm}(300 \times 300$ DPI $)$ 


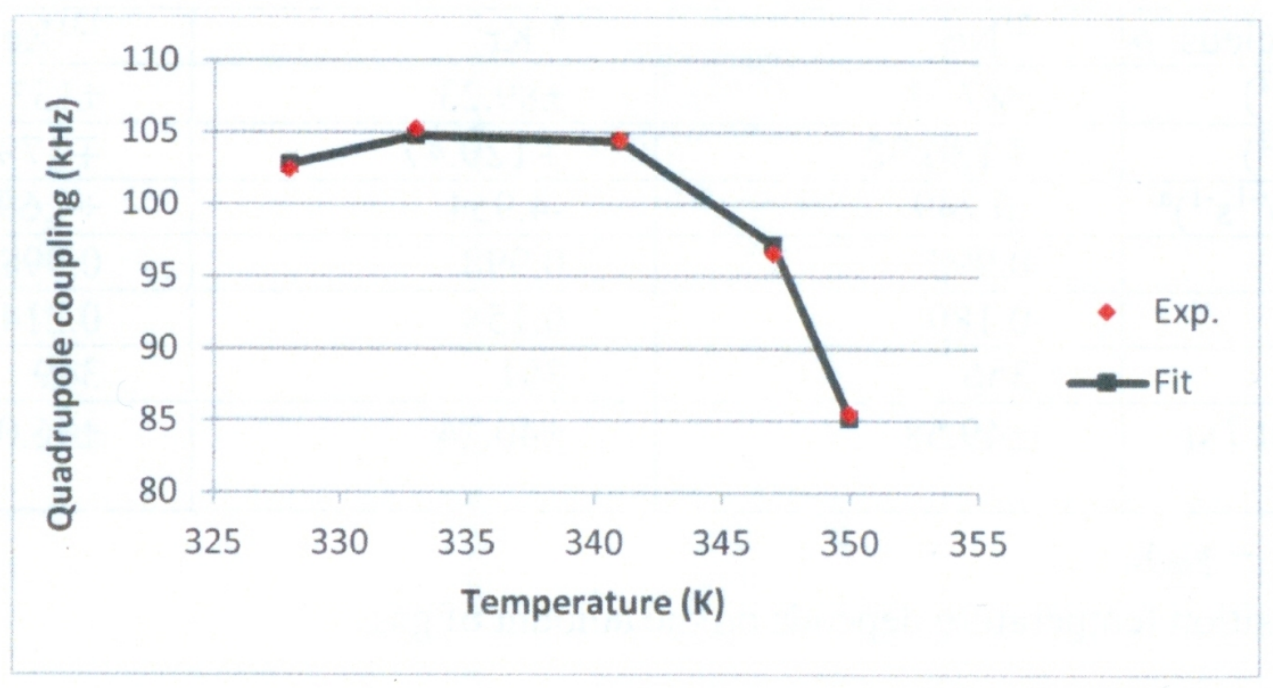

Magnitude of the $83 \mathrm{Kr}$ quadrupole coupling as a function of temperature in ZLI 1167 liquid crystal.

$119 \times 65 \mathrm{~mm}(300 \times 300$ DPI $)$ 


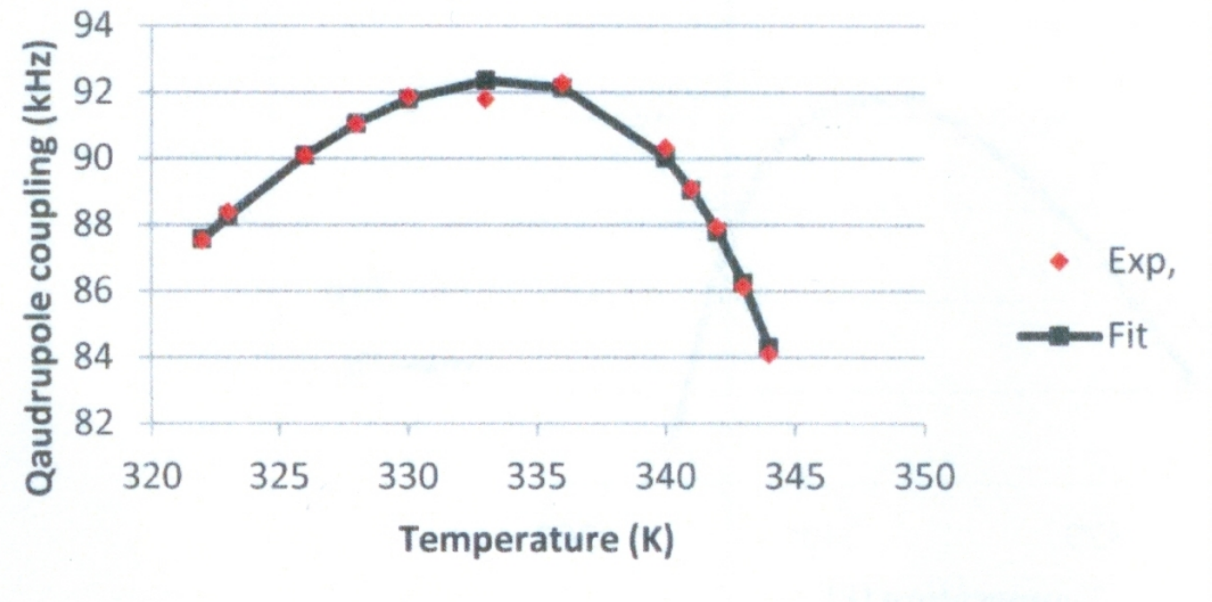

Magnitude of the 131 Xe quadrupole coupling as a function of temperature in ZLI 1167 liquid crystal. $113 \times 56 \mathrm{~mm}(300 \times 300 \mathrm{DPI})$ 
Orientational order parameter $\mathrm{S}$ as a function of reduced temperature T/TNI in ZLI 1167 as determined from the $21 \mathrm{Ne}, 83 \mathrm{Kr}$ and $131 \mathrm{Xe}$ NMR spectra.

$118 \times 68 \mathrm{~mm}(300 \times 300$ DPI $)$ 
129Xe and 131 Xe shielding (relative to xenon gas) as a function of temperature in FELIX-R\&D. Vertical lines indicate phase transition temperatures. One should note that the shieldings coincide in the isotropic phase and at the SmC-SmA phase transition point. The results are from experiments obtained on a $300 \mathrm{MHz}$ spectrometer $($ Bo $=7.05 \mathrm{~T})$. See Ref. [15].

$112 \times 75 \mathrm{~mm}(300 \times 300 \mathrm{DPI})$ 
Magnitude of the 131 Xe second order quadrupole shift (SOQS) as a function of temperature in the smectic C phase of FELIX-R\&D liquid crystal at Bo $=7.05 \mathrm{~T}$.

$$
104 \times 62 \mathrm{~mm}(300 \times 300 \mathrm{DPI})
$$


The separation of the satellite transitions determines the quadrupole coupling in the $131 \mathrm{Xe}$ NMR spectrum $134 \times 111 \mathrm{~mm}(300 \times 300 \mathrm{DPI})$ 


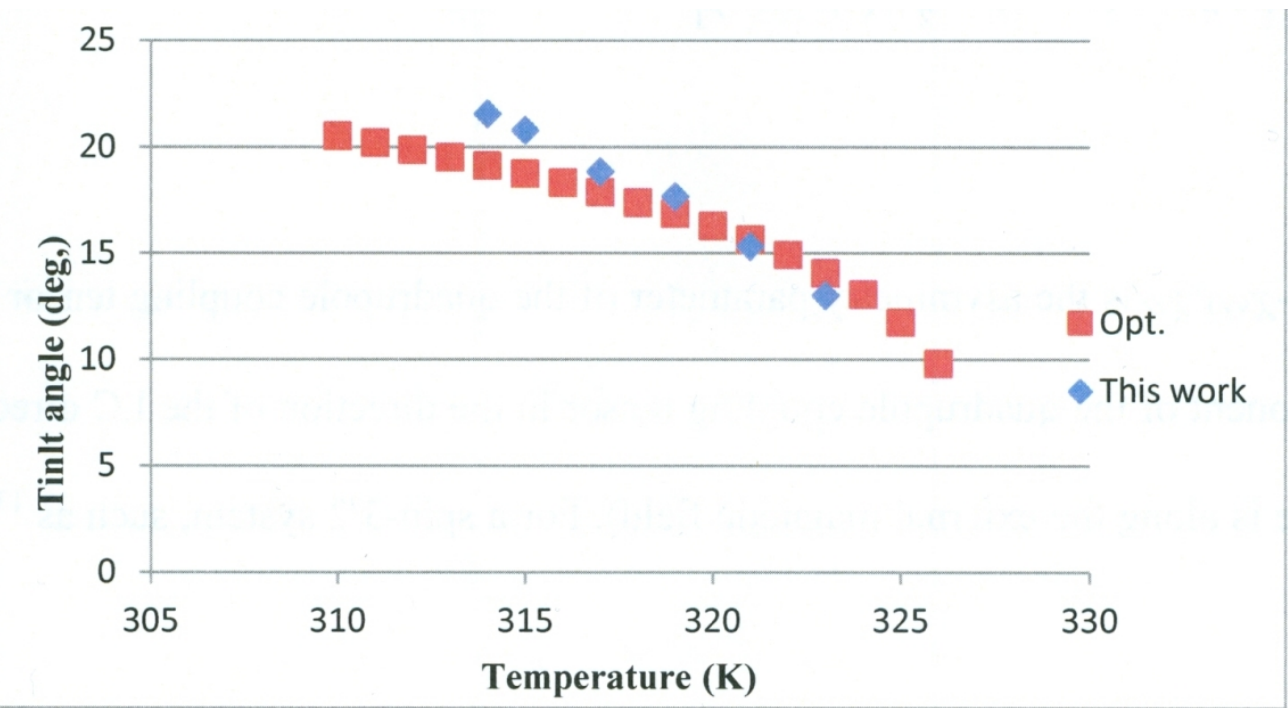

Comparison of the tilt angle values derived from the 131Xe SOQS using Eqn. (7) with those obtained by optical detection [17]. Note: The data reported in [17] are from pure liquid crystal while those of this work are from a sample with xenon gas.

$123 \times 66 \mathrm{~mm}(300 \times 300 \mathrm{DPI})$ 


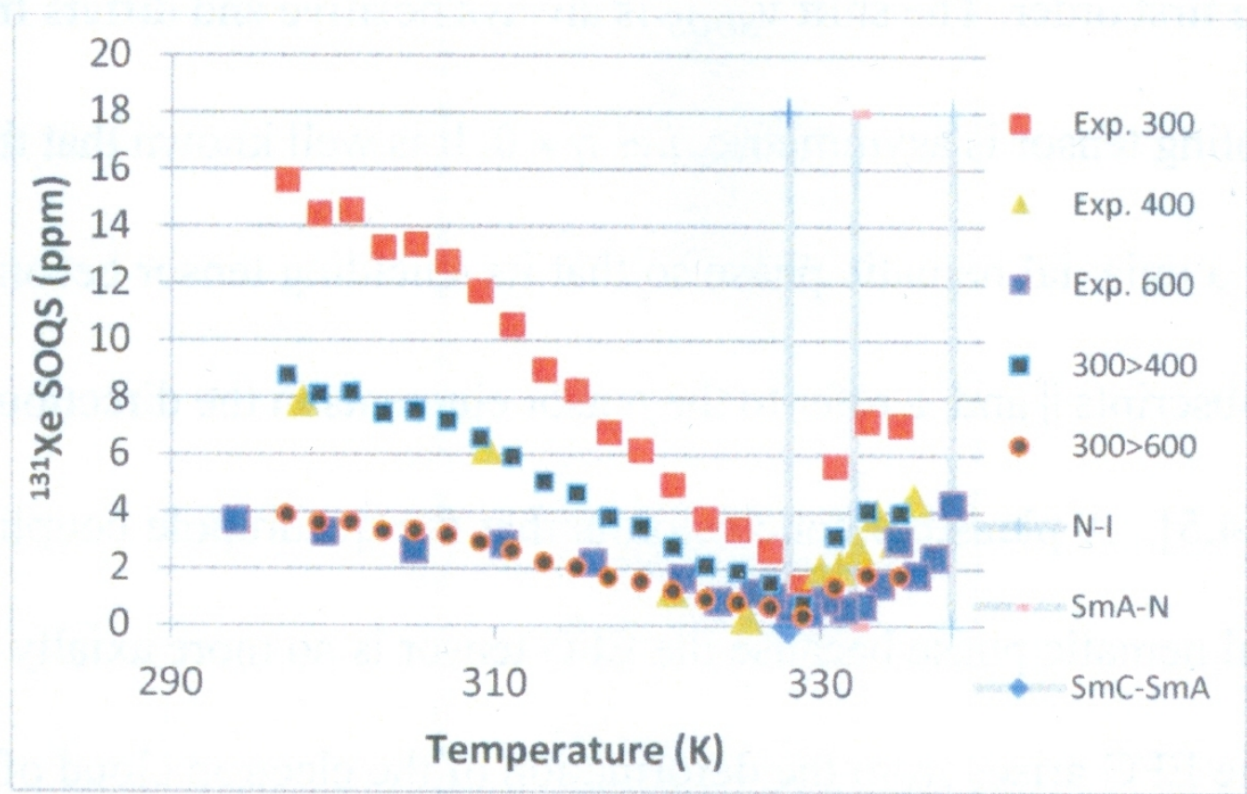

Magnitude of the 131Xe SOQS in FELIX-R\&D as a function of temperature at three magnetic fields corresponding to $1 \mathrm{H}$ resonance frequencies of 300,400 and $600 \mathrm{MHz}[15,18]$. Symbols $300>400$ and $300>600$ refer to scaling the $300 \mathrm{MHz}$ data to correspond to 400 and $600 \mathrm{MHz}$, respectively. Scaling factors are (300/400)2 and (300/600)2. Phase transition temperatures are indicated by vertical lines.

$105 \times 67 \mathrm{~mm}(300 \times 300 \mathrm{DPI})$ 


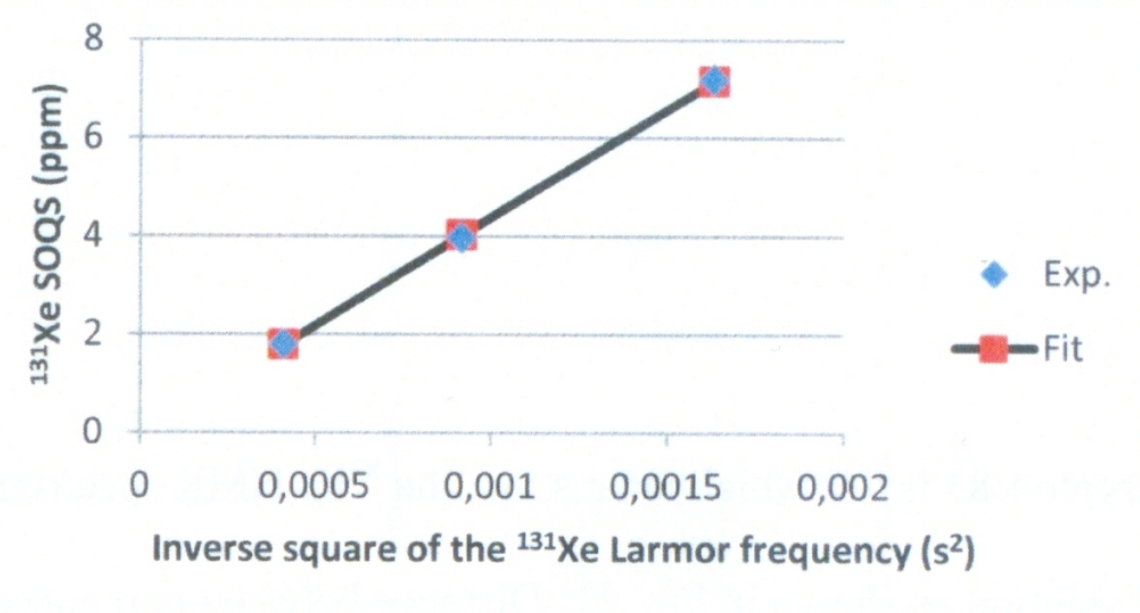

$131 \mathrm{Xe}$ SOQS as a function of the inverse square of the $131 \mathrm{Xe}$ Larmor frequency in the nematic phase of FELIX-R\&D at $334.8 \mathrm{~K}$.

$113 \times 59 \mathrm{~mm}(300 \times 300$ DPI $)$ 


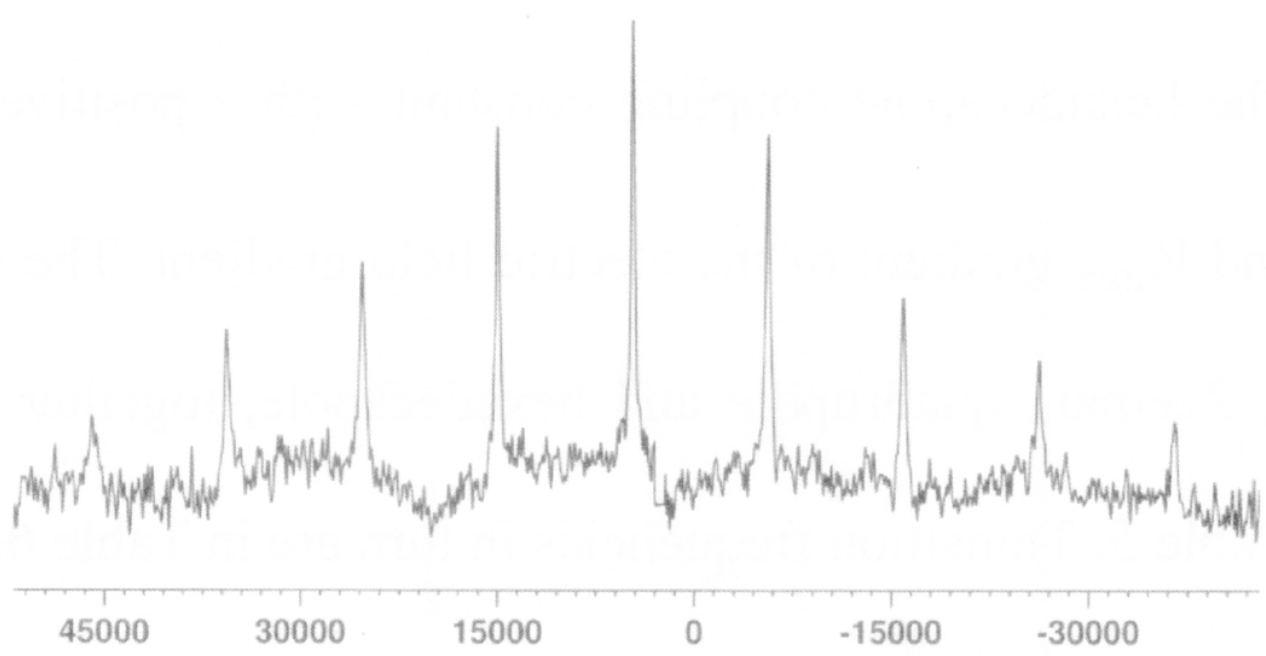

$83 \mathrm{Kr}$ NMR spectrum in a liquid-crystalline solution. The average distance between the consecutive peaks is $10300 \mathrm{~Hz}$ and thus the $83 \mathrm{Kr}$ quadrupole coupling is $247.2 \mathrm{kHz}$.

$91 \times 49 \mathrm{~mm}(300 \times 300 \mathrm{DPI})$ 
Table 1. NMR properties of quadrupolar noble gases and xenon-129.

\begin{tabular}{|c|c|c|c|c|c|c|c|}
\hline Nucleus & Spin & NA $\left.(\%)^{a}\right)$ & $\begin{array}{l}\gamma \\
\left(10^{7} \operatorname{rad} T^{-1} S^{-}\right)^{\mathrm{b})}\end{array}$ & $\mathrm{RR}^{\mathrm{c})}$ & $\begin{array}{l}\text { NMR freq. } \\
(\mathrm{MHz})^{\mathrm{d})}\end{array}$ & $\begin{array}{l}\mathrm{Q} \\
\left(10^{-31} \mathrm{~m}^{2}\right)^{\mathrm{e})}\end{array}$ & $\begin{array}{l}\text { Sternheimer } \\
\text { antishielding } \\
\text { factor } \gamma_{\infty} \text { f) }\end{array}$ \\
\hline${ }^{21} \mathrm{Ne}$ & $3 / 2$ & 0.257 & -2.113 & 0.0359 & 31.577 & $101.55(75)$ & -9.145 \\
\hline${ }^{83} \mathrm{Kr}$ & $9 / 2$ & 11.55 & -1.033 & 1.24 & 15.391 & $259(1)$ & -79.98 \\
\hline${ }^{129} \mathrm{Xe}$ & $1 / 2$ & 26.44 & -7.441 & 32.3 & 110.064 & - & - \\
\hline${ }^{131} \mathrm{Xe}$ & $3 / 2$ & 21.18 & 2.206 & 3.37 & 32.798 & $-114.6(1.1)$ & -168.5 \\
\hline
\end{tabular}

\footnotetext{
a) Natural abundance.

Gyromagnetic ratio.

Receptivity relative to ${ }^{13} \mathrm{C}$.

At $9.4 \mathrm{~T}$.

e) Nuclear quadrupole moment. Ref. [6].

f) Ref. $[7,8]$.
} 
Table 2. Results of the least-squares fits of function (1) to the experimental ${ }^{21} \mathrm{Ne},{ }^{83} \mathrm{Kr}$ and ${ }^{131} \mathrm{Xe}$ quadrupole couplings in ZLI 1167. Experiments do not reveal the sign of quadrupole couplings, and consequently the sign of the $A$ and $B$ factors but they do reveal their relative sign.

\begin{tabular}{|c|c|c|c|}
\hline Parameter $\vee$ Nucleus $\rightarrow$ & ${ }^{21} \mathrm{Ne}$ & ${ }^{83} \mathrm{Kr}$ & ${ }^{131} \mathrm{Xe}$ \\
\hline$A\left(10^{17} \mathrm{Vm}^{-2}\right)$ & \pm 87.31 & \pm 89.23 & \pm 133.25 \\
\hline$B\left(10^{17} \mathrm{Vm}^{-2}\right)$ & $\mp 136.90$ & $\mp 129.47$ & $\mp 179.25$ \\
\hline$C\left(10^{-13} \mathrm{~m}^{2} \mathrm{~V}^{-1} \mathrm{~s}^{-1}\right)^{\mathrm{a})}$ & -0.249 & -4.954 & +4.695 \\
\hline$y$ & 0.998 & 0.998 & 0.998 \\
\hline$z$ & 0.180 & 0.158 & 0.214 \\
\hline$T_{\mathrm{NI}}(\mathrm{K})^{\mathrm{b})}$ & 356 & 351 & 349 \\
\hline Total EFG at $T_{\mathrm{NI}}$ & \pm 49.58 & \pm 40.24 & \pm 46.01 \\
$\left(10^{17} \mathrm{Vm}^{-2}\right)$ & & & \\
\hline
\end{tabular}

a) $C=\frac{e Q}{h}\left(1-\gamma_{\infty}\right)$.

b) Phase transition temperature depends on the amount of gas. 
Table 3. Zeroth-order, first-order and second-order contribution to the resonance frequency of ${ }^{131} \mathrm{Xe}$ in a tilted liquid-crystalline phase $[15,18]$.

\begin{tabular}{lccc}
\hline Transition & $\begin{array}{c}\text { Frequency in } \\
\text { zeroth order }\end{array}$ & $\begin{array}{c}\text { First-order } \\
\text { correction }\end{array}$ & $\begin{array}{c}\text { Second-order } \\
\text { correction }\end{array}$ \\
\hline$-3 / 2 \ldots-1 / 2$ & $\boldsymbol{v}_{\mathrm{L}}$ & $\frac{\chi}{2} P_{2}(\cos \theta)$ & $\frac{3 \chi^{2}}{8 v_{L}}\left(1-\cos ^{2} \theta\right) \cos ^{2} \theta$ \\
$-1 / 2 \ldots+1 / 2$ & $\boldsymbol{v}_{\mathrm{L}}$ & - & $-\frac{3 \chi^{2}}{64 v_{L}}\left(1-\cos ^{2} \theta\right)\left(9 \cos ^{2} \theta-1\right)$ \\
$+1 / 2 \ldots+3 / 2$ & $\boldsymbol{v}_{\mathrm{L}}$ & $-\frac{\chi}{2} P_{2}(\cos \theta)$ & $\frac{3 \chi^{2}}{8 v_{L}}\left(1-\cos ^{2} \theta\right) \cos ^{2} \theta$ \\
\hline
\end{tabular}

a) $\quad=\frac{e Q\langle V\rangle}{h}$ is the quadrupole coupling and $P_{2}$ is the second order Legendre polynomial. 
Table 4. Transition frequencies up to the second order and relative intensities of ${ }^{131} \mathrm{Xe}$ NMR spectrum in a biaxial liquid crystal $[15,18]$.

\begin{tabular}{rcc}
\hline Transition & Frequency (in Hz) ${ }^{\text {a) }}$ & Relative intensity b) \\
\hline$-3 / 2 \leftrightarrow-1 / 2$ & $v_{L}+1 / 2 \chi_{z z}$ & 3 \\
$-1 / 2 \leftrightarrow+1 / 2$ & $v_{L}+\frac{1\left(\chi_{x x}-\chi_{y y}\right)^{2}}{48 n}=\nu_{L}+\frac{1\left(\eta \chi_{z z}\right)^{2}}{48 \quad n}$ & 4 \\
$+1 / 2 \leftrightarrow+3 / 2$ & $v_{L}-1 / 2 \chi_{z z}$ & 3 \\
\hline
\end{tabular}

a) $v_{L}=v_{o}(1-\sigma) \approx v_{o}$ where $v_{o}$ is the ${ }^{131} \mathrm{Xe}$ Larmor frequency.

b) Intensities are practically the same as in the first order. 
Table 5. Energy contributions (in $\mathrm{Hz}$ ) of various interactions to the spin states of ${ }^{83} \mathrm{Kr}$.

\begin{tabular}{|c|c|c|c|c|}
\hline $\mathrm{m}$ & $E_{z}{ }^{a)}$ & $\mathrm{E}_{\mathrm{Q}}^{\mathrm{b})}$ & $\left.E_{H}{ }^{c}\right)$ & $E^{(2) d)}$ \\
\hline $9 / 2$ & $-9 / 2 \boldsymbol{v}_{\mathrm{L}}$ & $36 \mathrm{~A}$ & $18 \mathrm{~B}$ & $-6 / 4 \mathrm{C}$ \\
\hline $7 / 2$ & $-7 / 2 \boldsymbol{v}_{\mathrm{L}}$ & $12 \mathrm{~A}$ & $-22 B$ & $-14 / 4 \mathrm{C}$ \\
\hline $5 / 2$ & $-5 / 2 v_{\mathrm{L}}$ & $-6 \mathrm{~A}$ & $-17 \mathrm{~B}$ & $-15 / 4 C$ \\
\hline $3 / 2$ & $-3 / 2 \boldsymbol{v}_{\mathrm{L}}$ & $-18 \mathrm{~A}$ & $3 B$ & $-11 / 4 \mathrm{C}$ \\
\hline $1 / 2$ & $-1 / 2 \boldsymbol{v}_{\mathrm{L}}$ & $-24 \mathrm{~A}$ & 18B & $-\mathrm{C}$ \\
\hline$-1 / 2$ & $1 / 2 \boldsymbol{v}_{\mathrm{L}}$ & $-24 \mathrm{~A}$ & $18 \mathrm{~B}$ & $\mathrm{C}$ \\
\hline$-3 / 2$ & $3 / 2 \boldsymbol{v}_{\mathrm{L}}$ & $-18 \mathrm{~A}$ & $3 \mathrm{~B}$ & $11 / 4 \mathrm{C}$ \\
\hline$-5 / 2$ & $5 / 2 \boldsymbol{v}_{\mathrm{L}}$ & $-6 \mathrm{~A}$ & $-17 \mathrm{~B}$ & $15 / 4 \mathrm{C}$ \\
\hline$-7 / 2$ & $7 / 2 \boldsymbol{v}_{\mathrm{L}}$ & $12 \mathrm{~A}$ & $-22 \mathrm{~B}$ & $14 / 4 \mathrm{C}$ \\
\hline$-9 / 2$ & $9 / 2 \boldsymbol{v}_{\mathrm{L}}$ & $36 \mathrm{~A}$ & $18 \mathrm{~B}$ & $6 / 4 \mathrm{C}$ \\
\hline
\end{tabular}

a) $\boldsymbol{v}_{\mathrm{L}}$ is the ${ }^{83} \mathrm{Kr}$ Larmor frequency,

b) $\mathrm{A}=\chi_{\mathrm{Q}} / 144$ and $\chi_{\mathrm{Q}}$ is the ${ }^{83} \mathrm{Kr}$ quadrupole coupling constant.

c) $\mathrm{B}=\chi_{\mathrm{H}} / 3456$ and $\chi_{\mathrm{H}}$ the ${ }^{83} \mathrm{Kr}$ hexadecapole coupling constant.

d) Second order correction. $\mathrm{C}=12\left(\chi_{\mathrm{Q}} \eta / 144\right)^{2} / \boldsymbol{v}_{\mathrm{L}}$ where $\eta$ is the asymmetry parameter of the ${ }^{83} \mathrm{Kr}$ quadrupole coupling tensor. Note: $\chi_{\mathrm{Q}} \eta=\chi_{\mathrm{xx}}-\chi_{\mathrm{yy}}$. 
Table 6. Frequencies and relative intensities of the single quantum transitions for ${ }^{83} \mathrm{Kr}$ in a liquidcrystalline solution. Frequency is the sum of the listed contributions.

\begin{tabular}{|cc|c|c|c|c|c|}
\hline Transition & No. & Zeeman term & $\begin{array}{c}\text { Quadrupole } \\
\text { term }\end{array}$ & $\begin{array}{c}\text { Hexadecapole } \\
\text { term }\end{array}$ & $\begin{array}{c}\text { Second } \\
\text { order } \\
\text { shift }\end{array}$ & $\begin{array}{c}\text { Relative } \\
\text { intensity }\end{array}$ \\
\hline $9 / 2 \ldots 7 / 2$ & 1 & $\boldsymbol{v}_{\mathrm{L}}$ & $-24 \mathrm{~A}$ & $-40 \mathrm{~B}$ & $-2 \mathrm{C}$ & 9 \\
\hline $7 / 2 \ldots 5 / 2$ & 2 & $\boldsymbol{v}_{\mathrm{L}}$ & $-18 \mathrm{~A}$ & $5 \mathrm{~B}$ & $-1 / 4 \mathrm{C}$ & 16 \\
\hline $5 / 2 \ldots 3 / 2$ & 3 & $\boldsymbol{v}_{\mathrm{L}}$ & $-12 \mathrm{~A}$ & $20 \mathrm{~B}$ & $\mathrm{C}$ & 21 \\
\hline $3 / 2 \ldots 1 / 2$ & 4 & $\boldsymbol{v}_{\mathrm{L}}$ & $-6 \mathrm{~A}$ & $15 \mathrm{~B}$ & $7 / 4 \mathrm{C}$ & 24 \\
\hline $1 / 2 \ldots-1 / 2$ & 5 & $\boldsymbol{v}_{\mathrm{L}}$ & 0 & 0 & $2 \mathrm{C}$ & 25 \\
\hline$-1 / 2 \ldots-3 / 2$ & 6 & $\boldsymbol{v}_{\mathrm{L}}$ & $6 \mathrm{~A}$ & $-15 \mathrm{~B}$ & $-7 / 4 \mathrm{C}$ & 24 \\
\hline$-3 / 2 \ldots-5 / 2$ & 7 & $\boldsymbol{v}_{\mathrm{L}}$ & $12 \mathrm{~A}$ & $-20 \mathrm{~B}$ & $\mathrm{C}$ & 21 \\
\hline$-5 / 2 \ldots-7 / 2$ & 8 & $\boldsymbol{v}_{\mathrm{L}}$ & $18 \mathrm{~A}$ & $-5 \mathrm{~B}$ & $-1 / 4 \mathrm{C}$ & 16 \\
\hline$-7 / 2 \ldots-9 / 2$ & 9 & $\boldsymbol{v}_{\mathrm{L}}$ & $24 \mathrm{~A}$ & $40 \mathrm{~B}$ & $-2 \mathrm{C}$ & 9 \\
\hline
\end{tabular}


Table 7. Differences of the frequencies of consecutive resonance lines. A, B and C are defined in the footnotes to Table 3 .

\begin{tabular}{|l|l|}
\hline Resonance lines & $\begin{array}{l}\text { Difference of the } \\
\text { frequencies }\end{array}$ \\
\hline $2-1$ & $6 \mathrm{~A}+45 \mathrm{~B}+7 / 4 \mathrm{C}$ \\
\hline $3-2$ & $6 \mathrm{~A}+15 \mathrm{~B}+5 / 4 \mathrm{C}$ \\
\hline $4-3$ & $6 \mathrm{~A}-5 \mathrm{~B}+3 / 4 \mathrm{C}$ \\
\hline $5-4$ & $6 \mathrm{~A}-15 \mathrm{~B}+1 / 4 \mathrm{C}$ \\
\hline $6-5$ & $6 \mathrm{~A}-15 \mathrm{~B}-15 / 4 \mathrm{C}$ \\
\hline $7-6$ & $6 \mathrm{~A}-5 \mathrm{~B}+11 / 4$ \\
\hline $8-7$ & $6 \mathrm{~A}+15 \mathrm{~B}-5 / 4 \mathrm{C}$ \\
\hline $9-8$ & $6 \mathrm{~A}+45 \mathrm{~B}-7 / 4 \mathrm{C}$ \\
\hline
\end{tabular}

